\title{
A nomogram for predicting survival in patients with breast cancer brain metastasis
}

\author{
ZHOU HUANG $^{1,2^{*}}$, BING SUN $^{1 *}$, SHIKAI WU ${ }^{1}$, XIANGYING MENG ${ }^{1}$, \\ YANG CONG ${ }^{1}$, GE SHEN $^{1}$ and SANTAI SONG ${ }^{3}$
}

\author{
${ }^{1}$ Department of Radiation Oncology, Affiliated Hospital of Academy of Military Medical Sciences, \\ Beijing 100071; ${ }^{2}$ Department of Radiation Oncology, National Cancer Center/Cancer Hospital, \\ Peking Union Medical College and Chinese Academy of Medical Sciences, Beijing 100021; ${ }^{3}$ Department of \\ Breast Cancer, Affiliated Hospital of Academy of Military Medical Sciences, Beijing 100071, P.R. China
}

Received February 7, 2017; Accepted January 18, 2018

DOI: $10.3892 / \mathrm{ol} .2018 .8259$

\begin{abstract}
Brain metastasis (BM) is common in patients with breast cancer. Predicting patient survival is critical for the clinical management of breast cancer brain metastasis (BCBM). The present study was designed to develop and evaluate a prognostic model for patients with newly diagnosed BCBM. Based on the clinical data of patients with BCBM treated in the Affiliated Hospital of Academy of Military Medical Sciences (Beijing, China) between 2002 and 2014, a nomogram was developed to predict survival using proportional hazards regression analysis. The model was validated internally by bootstrapping, and the concordance index (c-index) was calculated. A calibration curve and c-index were used to evaluate discriminatory and predictive ability, in order to compare the nomogram with widely used models, including recursive partitioning analysis (RPA), graded prognostic assessment (GPA) and breast-graded prognostic assessment (Breast-GPA). A total of 411 patients with BCBM were included in the development of this predictive model. The median overall survival time was 14.1 months. Statistically significant predictors for patient survival included biological subtype, Karnofsky performance score, leptomeningeal metastasis, extracranial metastasis, the number of brain metastases and disease-free survival. A nomogram for predicting 1- and 2-year overall survival rates was constructed, which exhibited good accuracy in predicting overall survival with a concordance index of 0.735. This model outperformed RPA, GPA and Breast-GPA, based on the comparisons of the c-indexes. The nomogram
\end{abstract}

Correspondence to: Dr Shikai Wu, Department of Radiation Oncology, Affiliated Hospital of Academy of Military Medical Sciences, 8 Dongda Street, Beijing 100071, P.R. China

E-mail: skywu4923@sina.com

${ }^{*}$ Contributed equally

Key words: brain metastasis, breast cancer, predictive model, nomogram constructed based on a multiple factor analysis was able to more accurately predict the individual survival probability of patients with BCBM, compared with existing models.

\section{Introduction}

Breast cancer is the leading cancer in women worldwide, in terms of incidence and cancer-associated mortality (1). The brain is one of the most common sites for breast cancer metastasis, with a rate of brain metastasis of $10-15 \%$ in patients with advanced breast cancer (2) and a rate of $30-55 \%$ in patients with HER-2 overexpression (3). The median survival time for patients with breast cancer brain metastasis (BCBM) ranges between 4 and 14 months (4-6).

In the era of personalized medicine, the use of the same treatments for all BCBM patients is no longer appropriate. The choice of treatment for a given patient depends upon numerous factors, including age, performance status and tumor characteristics such as breast biological subtypes, tumor site, number of brain metastases and extracranial metastasis. Considering that patients with BCBM are a heterogeneous group, it is necessary to introduce a simple breast cancer-specific prognostic index that may aid clinicians in selecting the appropriate treatment. Several prognostic models for patients with cancer have been developed and widely used in clinical oncology practice (7-9). For example, the Radiation Therapy Oncology Group established recursive partitioning analysis (RPA) in 1997 (7). The graded prognostic assessment (GPA) was constructed in 2008 and has been regarded as more accurate than RPA (8). Although RPA and GPA are widely used in the clinic, these were constructed on the basis of several different histological types of cancer and have limited use in breast cancer. In 2012, the Breast-GPA was developed based on analysis of the clinical features of 400 cases of BCBM (9).

Considering the limitations of RPA, GPA and Breast-GPA, there is a requirement for developing a novel prognostic model. A nomogram is a visual predictive tool based on statistical regression models, which measures the impact of various factors on the possibility of an event (10). This tool may aid clinicians in assessing patient risk of recurrence and prognosis, and in selecting appropriate patients for clinical trials. It has 
been demonstrated that a nomogram may improve predictive accuracy for clinical outcomes, compared with traditional prognostic models (11-17). The present study was designed to construct a novel prognostic model for BCBM using a nomogram approach. Furthermore, the present study also compared the novel model with existing PRA, GPA and Breast-GPA models, with the aim that the newly developed model would be useful in the treatment of patients with BCBM.

\section{Materials and methods}

Patients and treatment. The medical records of patients with BCBM, who had been admitted to the Affiliated Hospital of Academy of Military Medical Sciences (Beijing, China) between January 2002 and December 2014, were retrospectively analyzed. The diagnosis of breast cancer was pathologically confirmed, and brain metastasis was diagnosed by imaging or pathology. Patients who had more than one histological tumor type or missing data on key medical information were excluded from the present study. A total of 411 female patients with a median age of 47.6 years (range, 25.3-80.0 years) at brain metastases were finally included in present study. Based on the number and dimensions of brain metastases (BMs), these patients underwent local treatments, including surgical resection, whole brain radiotherapy (WBRT) and stereotactic radiosurgery (SRS). The most common regimen of WBRT was $40 \mathrm{~Gy}$ in 20 fractions and the most common regimen of SRS was $17 \mathrm{~Gy}$ in 1 fraction. Among the 411 patients with BCBM, $265(64.5 \%)$ were treated with WBRT with or without SRS, $188(45.7 \%)$ received SRS or surgical resection with or without WBRT, 154 (37.5\%) received WBRT only, and 69 (16.9\%) did not receive local treatment. For patients with fewer than three BMs, SRS was initially performed and WBRT was administered when the BM progressed or additional BMs developed.

Variables. In the present study, possible factors that affect $\mathrm{BM}$ prognosis were selected based on review of current literature $(9,18-21)$, including age, clinical stage, biological subtype, disease-free survival (DFS), occurrence time of BM, duration between diagnosis and BM, Karnofsky performance score (KPS) (22), extracranial metastasis, meningeal metastasis, symptoms of BM, and the number and size of BM lesions. The classification of biological subtypes was based on the 2011 St. Gallen International Expert Consensus (23). The response to treatment was evaluated using the Response Evaluation Criteria in Solid Tumors (RECIST 1.1) (24).

Data analysis, model construction and statistical analysis. Brain metastasis overall survival (BMOS) was defined as the duration between the diagnosis of BM and mortality or the end of follow-up. DFS was defined as the duration between surgery and the first recurrent metastasis. The database was closed on May 15, 2016. Univariate analysis and a multivariate Cox proportional hazards model were used to analyze the association between risk factors and survival. Variables that were significant in the univariate analysis were incorporated into the Cox proportional hazards model, and variable-independent prognostic factors were selected through backward stepwise analysis. $\mathrm{P}<0.05$ was considered to indicate a statistically significant difference.
The nomogram model was constructed on the foundation of the Cox proportional hazards model, and its performance was evaluated by internal validation with Bootstrap resampling (1,000 times), in order to minimize biases in the performance of the model $(10,25)$. Discrimination and calibration were used to assess nomogram performance. The discrimination ability (how well a model is able to distinguish between patients who succumbed to mortality and patients who survived.) of the nomogram was quantified by using the Harrell C-index (25). The c-index was similar to the area under the receiver operating characteristic (ROC) curve, with an index of 0.5 and 1 indicating the lack of concordance and perfect concordance, respectively (26). Calibration was obtained by plotting the calibrated curve of the association between the observed incidence and the predicted probabilities (27). The c-index was also used for the comparison of different models. The Kaplan-Meier method was used to plot the survival curves according to RPA, GPA and Breast-GPA prognosis models. The RPA model divides the patients into three different prognostic groups: group I (patients $<65$ years, KPS $\geq 70$, controlled primary tumor, and no extracranial metastasis), group II (all other patients not included in group I or III), and group III (KPS $<70)$. The GPA model divides the patients into four prognostic groups, according to the sum scores (GPA score 0-1, 1.5-2.5, 3.0 and 3.5-4) of four factors, including age, KPS, number of brain metastases, and extracranial metastasis. Furthermore, the Breast-GPA model also divides the patients into four prognostic groups, according to the sum scores (Breast-GPA score 0-1, 1.5-2.0, 2.5-3.0 and 3.5-4.0) of three prognostic factors: age, KPS and biological subtype. Statistical analyses were performed using SPSS 18.0 (SPSS, Inc., Chicago, IL, USA) and R software version 3.2.2 (http://www.r-project.org).

\section{Results}

Clinical features and survival. The characteristics of the selected patients are presented in Table I. The median follow-up time was 48.2 months. Cases of mortality, survival and loss to follow-up were 322 (78.3\%), 50 (12.2\%) and 39 (9.5\%), respectively. The median overall survival (OS) time following diagnosis of breast cancer was 68.2 months, while the median BMOS time was 14.1 months (range, 0.1-100.3 months), with 1-, 2- and 3-year survival rates of 55.9, 29.6 and $16.2 \%$, respectively. Furthermore, the median DFS time was 23.9 months (range, 0-232.3 months), the median duration between diagnosis of breast cancer and BM was 43.3 months (range, 0-353.2 months), and the median volume of brain metastases was $4.8 \mathrm{~cm}^{3}$ (range, $\left.0.1-139.7 \mathrm{~cm}^{3}\right)$.

\section{Nomogram model construction and validation}

Model construction. Univariate analysis results indicated that several factors, including molecular subtype, DFS, KPS, symptoms of BM, extracranial metastasis control, leptomeningeal metastasis and the number of BM lesions were associated with the survival of patients with BCBM (Table I). Furthermore, multivariate analysis results indicated that molecular type, KPS score, leptomeningeal metastasis, extracranial metastasis control, the number of BM lesions and DFS were independent factors that influenced the survival of patients with BCBM 
Table I. Patient characteristics.

\begin{tabular}{|c|c|c|c|}
\hline Characteristics & Number $(\%)$ & Median survival, months & P-value \\
\hline Age at BM, years & & & 0.318 \\
\hline$\leq 40$ & $105(25.5)$ & 11.1 & \\
\hline $40-60$ & $259(63.0)$ & 14.7 & \\
\hline$>60$ & $47(11.5)$ & 16.1 & \\
\hline Clinical stage & & & 0.828 \\
\hline I and II & $306(74.5)$ & 15.0 & \\
\hline III and IV & $105(25.5)$ & 12.3 & \\
\hline Biological subtype & & & $<0.001$ \\
\hline Luminal A & $140(34.0)$ & 14.7 & \\
\hline Luminal B & $87(21.2)$ & 20.2 & \\
\hline HER-2 Positive & $86(20.9)$ & 14.0 & \\
\hline Triple Negative & $94(22.9)$ & 8.7 & \\
\hline Unknown & $4(1.0)$ & - & \\
\hline DFS, months & & & 0.012 \\
\hline$>36$ & $125(30.4)$ & 17.1 & \\
\hline$\leq 36$ & $276(67.2)$ & 12.1 & \\
\hline Unknown & $10(2.4)$ & - & \\
\hline Diagnosis to BM, months & & & 0.072 \\
\hline$>44$ & $208(49.4)$ & 15.9 & \\
\hline$\leq 44$ & $203(50.6)$ & 11.6 & \\
\hline Symptoms of BM present & & & $<0.001$ \\
\hline Yes & $261(63.6)$ & 10.3 & \\
\hline No & $111(27.0)$ & 21.1 & \\
\hline Unknown & $39(9.5)$ & - & \\
\hline KPS & & & $<0.001$ \\
\hline$\geq 90$ & $169(41.1)$ & 19.3 & \\
\hline $70-90$ & $149(36.3)$ & 13.5 & \\
\hline$<70$ & $74(18.0)$ & 2.8 & \\
\hline Unknown & $19(4.6)$ & - & \\
\hline Extracranial metastasis control & & & $<0.001$ \\
\hline Controlled $(\mathrm{CR}+\mathrm{PR}+\mathrm{SD})$ & $162(39.4)$ & 18.5 & \\
\hline Uncontrolled (PD) & $227(55.2)$ & 9.8 & \\
\hline Unknown & $22(5.4)$ & - & \\
\hline Leptomeningeal metastasis & & & $<0.001$ \\
\hline Yes & $69(16.8)$ & 6.9 & \\
\hline No & $336(81.8)$ & 16.7 & \\
\hline Unknown & $6(1.5)$ & - & \\
\hline Number of BM lesions & & & $<0.001$ \\
\hline$\leq 3$ & $148(36.0)$ & 20.9 & \\
\hline$>3$ & $223(54.3)$ & 11.8 & \\
\hline Unknown & $40(9.7)$ & - & \\
\hline Total tumor volume, $\mathrm{cm}^{3}$ & & & 0.782 \\
\hline$\leq 4.8$ & $113(27.5)$ & 16.1 & \\
\hline$>4.8$ & $113(27.5)$ & 14.2 & \\
\hline Unknown & $185(45.0)$ & - & \\
\hline
\end{tabular}

BM, brain metastasis; DFS, disease-free survival; luminal A, ER/PR-positive and HER-2-negative; luminal B, ER/PR positive and HER-2-positive; HER-2, ER/PR-negative and HER-2-positive; Triple-negative ER/PR negative and HER-2-negative; KPS, Karnofsky performance score; CR, complete remission; PR, partial remission; SD, stable disease; PD, progressive disease; -, unknown. 
Table II. Multivariate analysis of prognostic factors.

\begin{tabular}{|c|c|c|c|c|c|}
\hline \multirow[b]{2}{*}{ Factor } & \multirow[b]{2}{*}{$\mathrm{b}$} & \multirow[b]{2}{*}{ P-value } & \multirow[b]{2}{*}{ HR } & \multicolumn{2}{|c|}{$95 \% \mathrm{CI}$} \\
\hline & & & & Lower & Upper \\
\hline Biological subtype & & $<0.001$ & & & \\
\hline Luminal B vs. A & -0.612 & $<0.001$ & 0.542 & 0.385 & 0.764 \\
\hline HER-2-positive vs. luminal A & -0.600 & 0.003 & 0.549 & 0.368 & 0.818 \\
\hline Triple negative vs. luminal A & -0.690 & $<0.001$ & 0.502 & 0.346 & 0.728 \\
\hline \multicolumn{6}{|l|}{ KPS } \\
\hline $70-80$ vs. $<70$ & 1.428 & $<0.001$ & 4.172 & 2.884 & 6.036 \\
\hline $90-100$ vs. $<70$ & 0.167 & 0.278 & 1.182 & 0.874 & 1.599 \\
\hline Leptomeningeal metastasis & -0.596 & 0.003 & 0.551 & 0.370 & 0.821 \\
\hline Extracranial metastasis control & 0.616 & $<0.001$ & 1.852 & 1.392 & 2.463 \\
\hline Number of brain metastases ( $\leq 3$ vs. $>3$ ) & 0.571 & $<0.001$ & 1.770 & 1.338 & 2.343 \\
\hline DFS (>36 vs. $\leq 36)$ & -0.312 & 0.039 & 0.732 & 0.544 & 0.985 \\
\hline
\end{tabular}

HR, hazard ratio; CI, confidence interval; KPS, Karnofsky performance score; extracranial disease control, $\geq \mathrm{SD}(\mathrm{CR}+\mathrm{PR}+\mathrm{SD})$ of extracranial diseases at the time of diagnosis of BM; luminal A, ER/PR-positive and HER-2-negative; luminal B, ER/PR positive and HER-2-positive; HER-2, ER/PR-negative and HER-2-positive; DFS, disease-free survival.

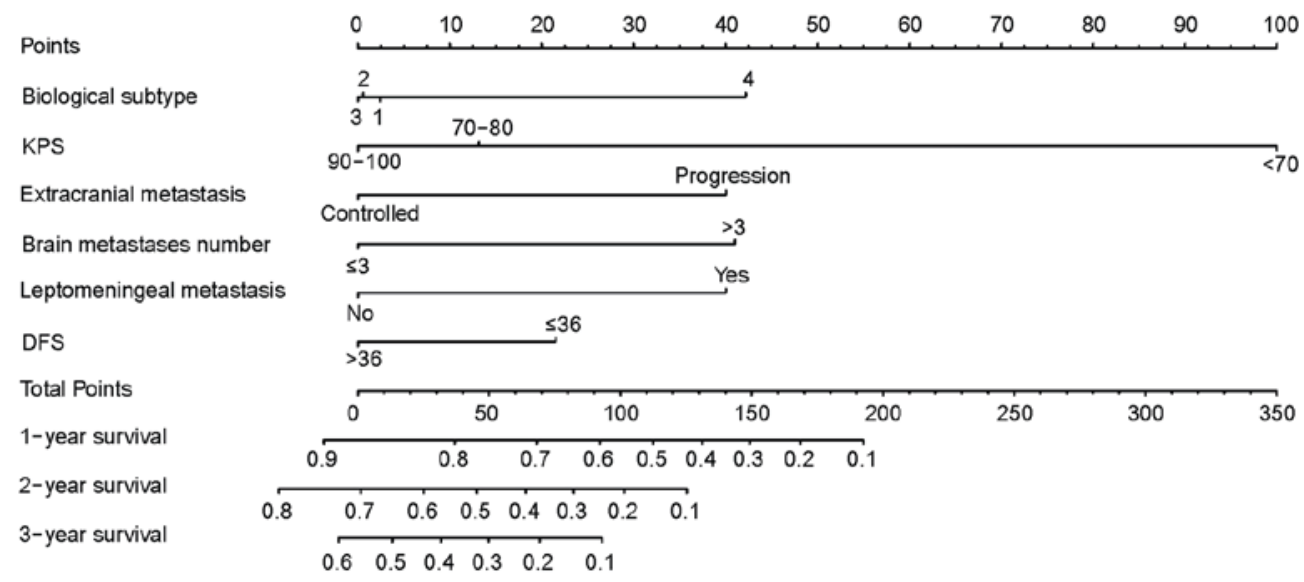

Figure 1. The nomogram for predicting overall survival was established for patients with breast cancer brain metastasis. The predictor points of each variable were observed on the upper-most point axis. There was a Total Points line at the bottom of the nomogram, and each variable score was summed to give the total points. A vertical line down to the 1-, 2- and 3-year survival lines allowed for the determination of probabilities for each individual. 1, luminal A; 2, luminal B; 3, HER-2 positive; 4, Triple negative; KPS, Karnofsky performance score (22); DFS, disease-free survival.

(Table II). The nomogram prognostic evaluation model was constructed based on the multivariate analysis (Fig. 1).

Model validation. The present study employed the bootstrap resampling method for the internal validation of the model to reduce the over-fitting of the model. The c-index was 0.73 (95\% CI, 0.70-0.77), indicating a good discrimination. The calibration plot of 1- and 2-year OS rates revealed a good agreement between observed values and predicted values (Fig. 2).

As shown in Fig. 3, there was overlapping between groups I and II in the RPA model (Fig. 3A; P=0.091). In addition, there was overlapping between groups II, III and IV in the using the GPA model (Fig. 3B; P=0.103). Furthermore, the discrimination was not satisfactory between groups II and III in Breast-GPA model (Fig. 3C; $\mathrm{P}=0.213$ ). Based on the results of the survival curves and P-values, it was concluded that the three aforementioned prognosis models were not satisfactory for differentiating patients with different survival times. The c-indexes were $0.73,0.64,0.61$ and 0.63 for the nomogram, Breast-GPA, GPA and RPA, respectively (Table III).

\section{Discussion}

Brain metastasis significantly impacts the prognosis and quality of life of patients with breast cancer. Precise prognostic predication contributes not only to the selection of a suitable therapeutic regimen, but also to the selection of appropriate patients for clinical trials. The present study developed a novel nomogram model for prognosis prediction through the 

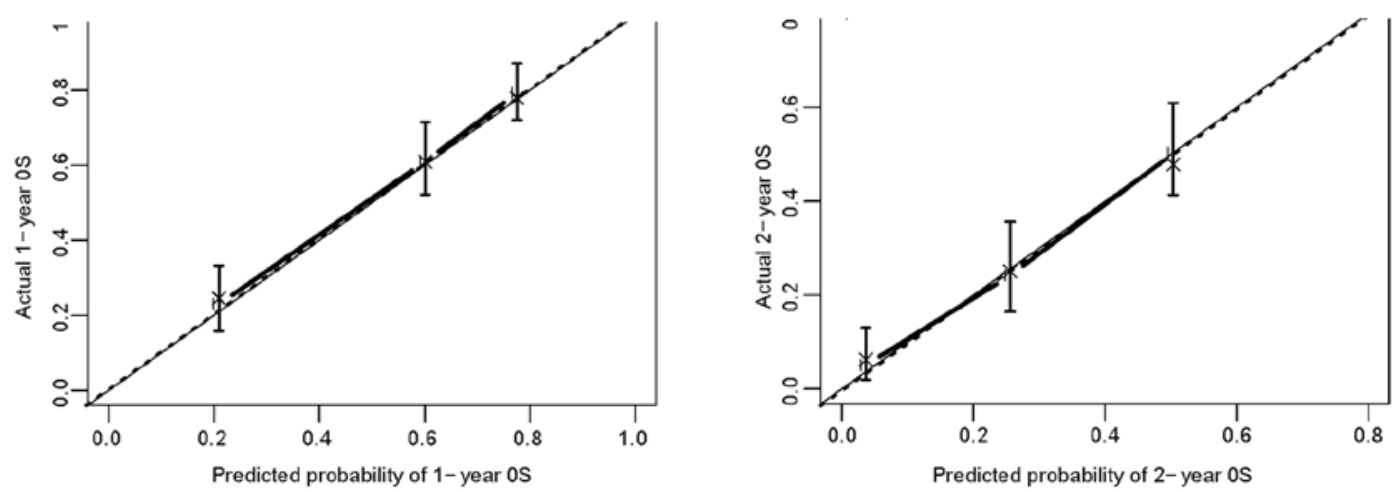

Figure 2. The calibration plots to predict the 1-year and 2-year OS rates for all patients. The x-axis represents the predicted OS and the y-axis represents the actual OS. The 45-degree line indicated an ideal reference line, which implies that the predicted OS rates were equal to the observed results. OS, overall survival.
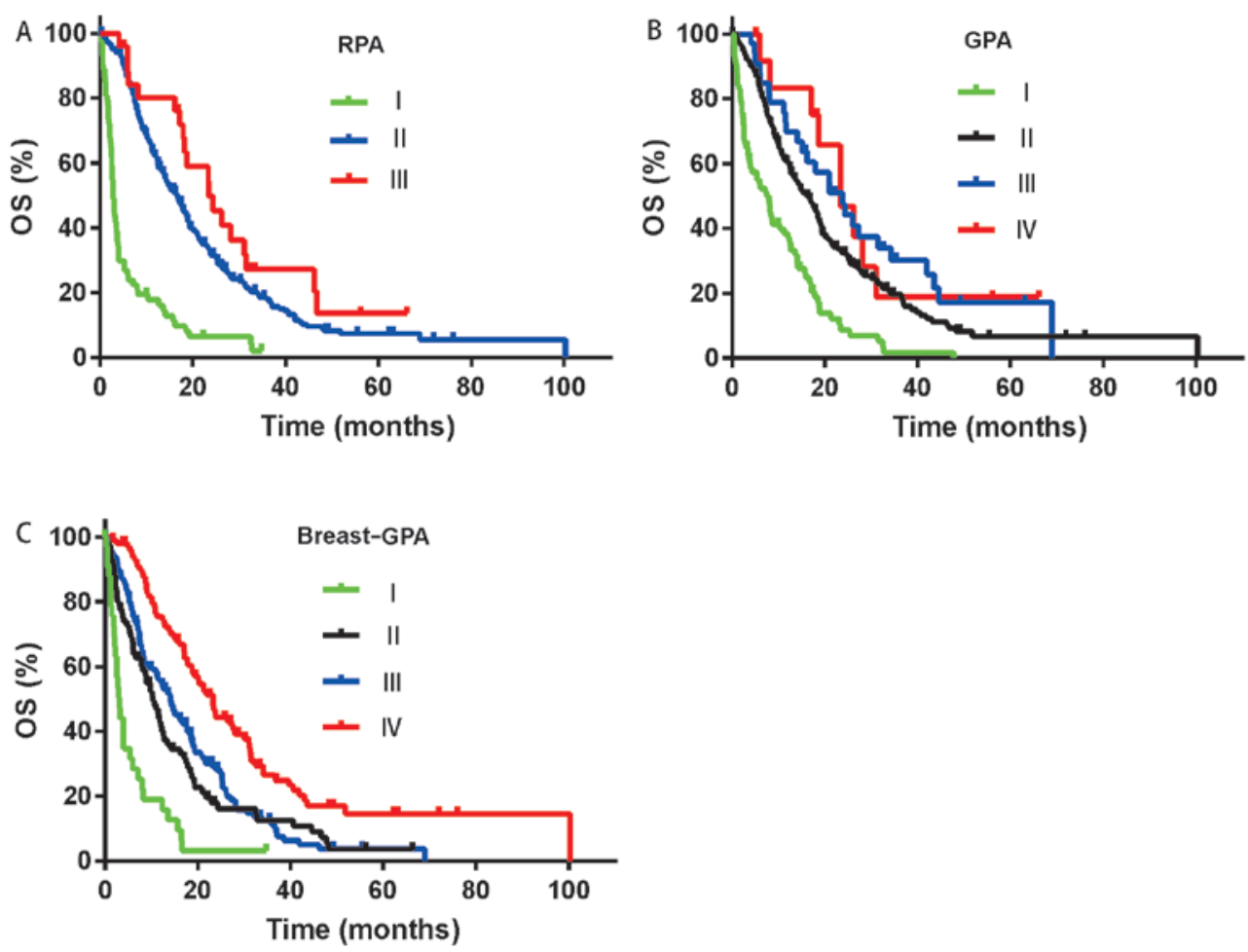

Figure 3. Overall survival of 411 patients according to the (A) RPA, (B) GPA and (C) Breast-GPA models. RPA, recursive partitioning analysis; GPA, graded prognostic assessment.

evaluation of several prognostic factors in a relatively large group of patients with BCBM.

The median survival the of the patients with BCBM enrolled in the present study was 14.1 months, with a 1-year survival rate of $56.5 \%$, which was slightly higher than that reported previously $(9,28,29)$. This may be associated with the recent advances in the diagnosis and treatment of brain tumors, particularly in the application of targeted therapies in patients with BCBM. Targeted therapeutic agents, including trastuzumab, lapatinib and pertuzumab have become the standard treatment for patients with HER-2 overexpression. In the present study, up to $85.5 \%$ of patients with HER-2 overexpression received anti-HER-2 therapy, with a median survival time of 17.9 months, which was similar to that reported previously (range, 11.6-19.5 months) (30,31).
The primary purpose of developing a prognostic model is to guide clinical treatment. Therefore, it would be better to exclude therapeutic and subjective factors when selecting prognostic factors $(32,33)$. Prognostic models used previously or currently in clinic often lack the evaluation of tumor biology factors, including tumor volume, meningeal metastases, molecular types and symptoms of BM (7-9), which are the influencing factors of prognosis $(34,35)$ Therefore, these models failed to accurately predict patient survival. In order to overcome the weaknesses of these models, the present study used univariate and multivariate analyses to identify factors that influence patient survival. It was revealed that molecular subtypes, KPS, extracranial control, leptomeningeal metastasis, number of BM lesions and DFS were independent 
Table III. Comparison of the nomogram with different predictive models.

\begin{tabular}{lccc}
\hline & & \multicolumn{2}{c}{$95 \%$ CI } \\
\cline { 3 - 4 } Method & C-index & Lower & Upper \\
\hline Nomogram & 0.735 & 0.703 & 0.767 \\
RPA & 0.633 & 0.603 & 0.662 \\
GPA & 0.614 & 0.583 & 0.646 \\
Breast-GPA & 0.640 & 0.609 & 0.671 \\
\hline
\end{tabular}

CI, confidence interval; RPA, recursive partitioning analysis; GPA, graded prognostic assessment.

factors for the prognosis of patients with BCBM. Patients with leptomeningeal metastasis were generally excluded in published studies. However, literature and clinical experience indicated that meningeal metastasis is one of the major factors of poor prognosis in patients with BCBM (36). Therefore, the present study included the clinical conditions of patients in the development of the model; and multivariate analysis results revealed that meningeal metastasis was an independent factor for patient prognosis. There were 69 (16.8\%) cases of meningeal metastases among the patients enrolled in the present study, which was slightly more than the numbers reported in previous studies $(37,38)$. This may be associated with the extended survival of patients, as well as the wide application of magnetic resonance imaging in the diagnosis of brain tumors.

A nomogram is able to assess patient survival time, which is beneficial for individualized therapy. The existing RPA, GPA and Breast-GPA models simply divide these patients into several subgroups, with great difference existing within the same subgroups (39). The present study compared different models using the c-index and survival curves, and demonstrated that the novel nomogram was superior to existing prognostic models (RPA, GPA and Breast-GPA). Furthermore, a crossover of survival curves among different groups in the RPA, GPA and Breast-GPA models was observed, which may be associated with the lack of molecular indices of breast cancer, inconsistent pathological types, differences in patient grouping, the selection of different prognostic factors, as well as defects in the modeling methods of RPA and GPA. Although the RPA model was constructed based on the results of 1,200 cases of BM, there were only 137 (12\%) cases of breast cancer (7). The GPA model was based on the analysis of 1,960 cases, but only 222 (11\%) cases of breast cancer were included (8).

Among the 411 patients included in the present study, $74.5 \%$ would be diagnosed with grade II disease based on the RPA model, and the median survival time of patients with grade II disease was 16.7 months (range, 0.2-100.3 months). This indicated the significantly different survival times within the same group. This discrepancy may result in administering palliative treatment to patients who should receive active treatment.

Although the nomogram model developed in the present study exhibited a good predictive ability, certain shortcomings remained. For example, as is often the case with retrospective studies, certain patient information was not available and therefore, bias was inevitable. Although the sample size was relatively large, the study population was selected from one hospital. Furthermore, it requires validation in other research institutions.

In conclusion, the present study developed and validated a nomogram prognosis evaluation model for patients with BCBM, which was demonstrated to be improved compared with the presently used RPA, GPA and Breast-GPA models. This model may be used to guide individual treatments and in selecting an appropriate patient population for clinical trials.

\section{Acknowledgements}

Not applicable.

\section{Funding}

No funding was obtained for the present study.

\section{Availability of data and materials}

All data are fully available upon request.

\section{Author's contributions}

Conception and design were undertaken by SKW. Collection of patient information and drafting of the article was undertaken by ZH. Data interpretation was performed by BS and ZH. XYM, YC, GS and STS participated in patient treatment, and helped revising the manuscript. All authors read and approved the final manuscript.

\section{Ethical approval and consent to participate}

All procedures involving human participants were performed in accordance with the ethical standards of the Affiliated Hospital of Academy of Military Medical Sciences and China Research Committee and with the 1964 Declaration of Helsinki and its later amendments or comparable ethical standards. Written informed consent was obtained from all participants included in the present study.

\section{Consent for publication}

Not applicable.

\section{Competing interests}

The authors declare that there are no competing interests.

\section{References}

1. Torre LA, Bray F, Siegel RL, Ferlay J, Lortet-Tieulent J and Jemal A: Global cancer statistics, 2012. CA Cancer J Clin 65: 87-108, 2015.

2. Barnholtz-Sloan JS, Sloan AE, Davis FG, Vigneau FD, Lai P and Sawaya RE: Incidence proportions of brain metastases in patients diagnosed (1973 to 2001) in the metropolitan detroit cancer surveillance system. J Clin Oncol 22: 2865-2872, 2004.

3. Lin NU, Amiri-Kordestani L, Palmieri D, Liewehr DJ and Steeg PS: CNS metastases in breast cancer: Old challenge, new frontiers. Clin Cancer Res 19: 6404-6418, 2013. 
4. DiStefano A, Yong Yap Y, Hortobagyi GN and Blumenschein GR: The natural history of breast cancer patients with brain metastases. Cancer 44: 1913-1918, 1979.

5. Tabouret E, Metellus P, Goncalves A, Esterni B, Charaffe-Jauffret E, Viens P and Tallet A: Assessment of prognostic scores in brain metastases from breast cancer. Neuro Oncol 16: 421-428, 2014.

6. Altundag K, Bondy ML, Mirza NQ, Kau SW, Broglio K, Hortobagyi GN and Rivera E: Clinicopathologic characteristics and prognostic factors in 420 metastatic breast cancer patients with central nervous system metastasis. Cancer 110: 2640-2647, 2007.

7. Gaspar L, Scott C, Rotman M, Asbell S, Phillips T, Wasserman T, McKenna WG and Byhardt R: Recursive partitioning analysis (RPA) of prognostic factors in three radiation therapy oncology group (RTOG) brain metastases trials. Int J Radiat Oncol Biol Phys 37: 745-751, 1997.

8. Sperduto PW, Berkey B, Gaspar LE, Mehta M and Curran W: A new prognostic index and comparison to three other indices for patients with brain metastases: An analysis of 1,960 patients in the RTOG database. Int J Radiat Oncol Biol Phys 70: 510-514, 2008.

9. Sperduto PW, Kased N, Roberge D, Xu Z, Shanley R, Luo X, Sneed PK, Chao ST, Weil RJ, Suh J, et al: Effect of tumor subtype on survival and the graded prognostic assessment for patients with breast cancer and brain metastases. Int J Radiat Oncol Biol Phys 82: 2111-2117, 2012.

10. Iasonos A, Schrag D, Raj GV and Panageas KS: How to build and interpret a nomogram for cancer prognosis. J Clin Oncol 26: 1364-1370, 2008.

11. Pietrantonio F, Aprile G, Rimassa L, Franco P, Lonardi S, Cremolini C, Biondani P, Sbicego EL, Pasqualetti F, Tomasello G, et al: A new nomogram for estimating survival in patients with brain metastases secondary to colorectal cancer. Radiother Oncol 117: 315-321, 2015.

12. Graesslin O, Abdulkarim BS, Coutant C, Huguet F, Gabos Z, Hsu L, Marpeau O, Uzan S, Pusztai L, Strom EA, et al: Nomogram to predict subsequent brain metastasis in patients with metastatic breast cancer. J Clin Oncol 28 : 2032-2037, 2010

13. Ahn HK, Lee S, Park YH, Sohn JH, Jo JC, Ahn JH, Jung KH, Park S, Cho EY, Lee JI, et al: Prediction of outcomes for patients with brain parenchymal metastases from breast cancer (BC): A new BC-specific prognostic model and a nomogram. Neuro Oncol 14: 1105-1113,2012.

14. Sternberg CN: Are nomograms better than currently available stage groupings for bladder cancer? J Clin Oncol 24: 3819-3820, 2006.

15. Marko NF, Xu Z, Gao T, Kattan MW and Weil RJ: Predicting survival in women with breast cancer and brain metastasis: A nomogram outperforms current survival prediction models. Cancer 118: 3749-3757, 2012

16. Yang Y, Zhang YJ, Zhu Y, Cao JZ, Yuan ZY, Xu LM, Wu JX Wang $\mathrm{W}, \mathrm{Wu} \mathrm{T}, \mathrm{Lu} \mathrm{B}$, et al: Prognostic nomogram for overall survival in previously untreated patients with extranodal NK/T-cell lymphoma, nasal-type: A multicenter study. Leukemia 29: 1571-1577, 2015

17. Tang LQ, Li CF, Li J, Chen WH, Chen QY, Yuan LX, Lai XP, $\mathrm{He}$ Y, Xu YX, Hu DP, et al: Establishment and validation of prognostic nomograms for endemic nasopharyngeal carcinoma. J Natl Cancer Inst 108: pii: djv291, 2015.

18. Subbiah IM, Lei X, Weinberg JS, Sulman EP, Chavez-MacGregor M, Tripathy D, Gupta R, Varma A, Chouhan J, Guevarra RP, et al: Validation and development of a modified breast graded prognostic assessment as a tool for survival in patients with breast cancer and brain metastases. J Clin Oncol 33: 2239-2245, 2015 .

19. Dawood S, Gonzalez-Angulo AM, Albarracin C, Yu TK, Hortobagyi GN, Buchholz TA and Woodward WA: Prognostic factors of survival in the trastuzumab era among women with breast cancer and brain metastases who receive whole brain radiotherapy: A single-institution review. Cancer 116: 3084-3092, 2010

20. Kased N, Binder DK, McDermott MW, Nakamura JL, Huang K, Berger MS, Wara WM and Sneed PK: Gamma Knife radiosurgery for brain metastases from primary breast cancer. Int J Radiat Oncol Biol Phys 75: 1132-1140, 2009.

21. Le Scodan R, Massard C, Jouanneau L, Coussy F, Gutierrez M, Kirova Y, Lerebours F, Labib A and Mouret-Fourme E: Brain metastases from breast cancer: Proposition of new prognostic score including molecular subtypes and treatment. J Neurooncol 106: 169-176, 2012.
22. Schag CC, Heinrich RL and Ganz PA: Karnofsky performance status revisited: Reliability, validity, and guidelines. J Clin Oncol 2: 187-193, 1984

23. Goldhirsch A, Wood WC, Coates AS, Gelber RD, Thürlimann B and Senn HJ; Panel members: Strategies for subtypes-dealing with the diversity of breast cancer: Highlights of the St Gallen international expert consensus on the primary therapy of early breast cancer 2011. Ann Oncol 22: 1736-1747, 2011.

24. Eisenhauer EA, Therasse P, Bogaerts J, Schwartz LH, Sargent D, Ford R, Dancey J, Arbuck S, Gwyther S, Mooney M, et al: New response evaluation criteria in solid tumours: Revised RECIST guideline (version 1.1). Eur J Cancer 45: 228-247, 2009.

25. Harrell FE Jr, Lee KL and Mark DB: Multivariable prognostic models: Issues in developing models, evaluating assumptions and adequacy, and measuring and reducing errors. Stat Med 15: 361-387, 1996.

26. Harrell FE Jr, Lee KL, Califf RM, Pryor DB and Rosati RA: Regression modelling strategies for improved prognostic prediction. Stat Med 3: 143-152, 1984.

27. Coutant C, Olivier C, Lambaudie E, Fondrinier E, Marchal F, Guillemin F, Seince N, Thomas V, Levêque J, Barranger E, et al: Comparison of models to predict nonsentinel lymph node status in breast cancer patients with metastatic sentinel lymph nodes: A prospective multicenter study. J Clin Oncol 27: 2800-2808, 2009.

28. Niwinska A and Murawska M: New breast cancer recursive partitioning analysis prognostic index in patients with newly diagnosed brain metastases. Int J Radiat Oncol Biol Phys 82: 2065-2071, 2012

29. Kim HJ, Im SA, Keam B, Kim YJ, Han SW, Kim TM, Oh DY, Kim JH, Lee SH, Chie EK, et al: Clinical outcome of central nervous system metastases from breast cancer: Differences in survival depending on systemic treatment. J Neurooncol 106: 303-313, 2012.

30. Dawood S, Broglio K, Esteva FJ, Ibrahim NK, Kau SW, Islam R, Aldape KD, Yu TK, Hortobagyi GN and Gonzalez-Angulo AM: Defining prognosis for women with breast cancer and CNS metastases by HER2 status. Ann Oncol 19: 1242-1248, 2008

31. Le Scodan R, Jouanneau L, Massard C, Gutierrez M, Kirova Y, Cherel P, Gachet J, Labib A and Mouret-Fourme E: Brain metastases from breast cancer: Prognostic significance of HER-2 overexpression, effect of trastuzumab and cause of death. BMC Cancer 11: 395, 2011.

32. Lagerwaard FJ, Levendag PC, Nowak PJ, Eijkenboom WM, Hanssens PE and Schmitz PI: Identification of prognostic factors in patients with brain metastases: A review of 1292 patients. Int J Radiat Oncol Biol Phys 43: 795-803, 1999.

33. Balachandran VP, Gonen M, Smith JJ and DeMatteo RP: Nomograms in oncology: More than meets the eye. Lancet Oncol 16: e173-e180, 2015.

34. Chamberlain MC: Leptomeningeal metastases in the MRI era Neurology 76: 200; author reply 200-201, 2011.

35. Kondziolka D, Kano H, Harrison GL, Yang HC, Liew DN, Niranjan A, Brufsky AM, Flickinger JC and Lunsford LD: Stereotactic radiosurgery as primary and salvage treatment for brain metastases from breast cancer. Clinical article. J Neurosurg 114: 792-800, 2011.

36. Song WG, Wang YF, Wang RL, Qu YE, Zhang Z, Li GZ, Xiao Y, Fang $\mathrm{F}$ and $\mathrm{Chen} \mathrm{H}$ : Therapeutic regimens and prognostic factors of brain metastatic cancers. Asian Pac J Cancer Prev 14: 923-927, 2013.

37. Brower JV, Saha S, Rosenberg SA, Hullett CR and Ian Robins H: Management of leptomeningeal metastases: Prognostic factors and associated outcomes. J Clin Neurosci 27: 130-137, 2016.

38. Dayan A, Koca D, Akman T, Oztop I, Ellidokuz H and Yilmaz U: The factors that have an impact on the development of brain metastasis in the patients with breast cancer. J Cancer Res Ther 8: 542-548, 2012

39. Braccini AL, Azria D, Thezenas S, Romieu G, Ferreri JM and Jacot W: Comparative performances of prognostic indexes for breast cancer patients presenting with brain metastases. BMC Cancer 13: 70, 2013

This work is licensed under a Creative Commons Attribution-NonCommercial-NoDerivatives 4.0 International (CC BY-NC-ND 4.0) License. 